\title{
Tri-Modality Small Animal Imaging System
}

\author{
B. K. Kundu, A. V. Stolin, Student Member, IEEE, J. Pole, Student Member, IEEE, L. Baumgart, M. Fontaine, \\ R. Wojcik, B. Kross, Carl Zorn, S. Majewski, and M. B. Williams, Member, IEEE
}

\begin{abstract}
Our group is developing a scanner that combines $x$-ray, single gamma, and optical imaging on the same rotating gantry. Two functional modalities (SPECT and optical) are included because they have different strengths and weaknesses in terms of spatial and temporal decay lengths in the context of in vivo imaging, and because of the recent advent of multiple reporter gene constructs. The effect of attenuation by biological tissue on the detected intensity of the emitted signal was measured for both gamma and optical imaging. Attenuation by biological tissue was quantified for both the bioluminescent emission of luciferace and for the emission light of the near infrared fluorophore cyanine 5.5, using a fixed excitation light intensity. Experiments were performed to test the feasibility of using either single gamma or $x$-ray imaging to make depth-dependent corrections to the measured optical signal. Our results suggest that significant improvements in quantitation of optical emission are possible using straightforward correction techniques based on information from other modalities. Development of an integrated scanner in which data from each modality are obtained with the animal in a common configuration will greatly simplify this process.
\end{abstract}

\section{INTRODUCTION}

$\mathbf{T}$ HE USE OF multiple, complementary imaging modalities can greatly enhance the information obtained in an in-vivo animal study. It is often desirable to obtain such data in quick succession if not simultaneously because of the time evolution of biologic processes in the animal. Additionally, it is also desirable to have the animal in a fixed configuration to permit image co-registration and fusion. We are developing a compact scanner, which will combine $\mathrm{x}$-ray, gamma, and bioluminescence/fluorescence optical imaging on the same rotating gantry. X-ray images will provide anatomic information, whereas gamma and optical images will provide functional information [1]. Two functional modalities are being included because they have very different strengths and weaknesses in the context of in vivo imaging. First, the majority of detected photons in single gamma imaging travel straight paths from the decaying isotope atom to the camera. Therefore the resolution of the source emitters can be as good as the spatial resolution of the camera ( $\sim 1 \mathrm{~mm}$ or less). On the other hand, in optical imaging the vast majority of photons have undergone many scattering interactions before reaching the surface of the animal. Thus the spatial distribution of the photons escaping from the surface is not a good approximation of the spatial distribution of the

Manuscript received November 15, 2004. This work was supported by The National Institute of Health under Grant R01 CA69452, and in part by NIH/NCI Grant 5 R24 CA092871-04 (M. Pomper, Johns Hopkins University).

B. K. Kundu, A. V. Stolin, J. Pole, and M. B. Williams are with the Department of Radiology, University of Virginia, Charlottesville, VA 22904 USA (e-mail: bkk5a@virginia.edu).

R. Wocjik, B. Kross, C. Zorn, and S. Majewski are with the Thomas Jefferson National Accelerator Facility, Newport News, VA 23606 USA.

Digital Object Identifier 10.1109/TNS.2005.862970 emitters unless the sources are located on the animal's surface. Second, optical probes are typically capable of multiple photon emission from a single molecule, whereas gamma probes are typically labeled with a single radioisotope atom. For example, the bioluminescent reporter firefly luciferace emits light as long as it is in the presence of a catalyst (luciferine) and energy source (ATP). Fluorescent reporters emit light as long as they are exposed to particular excitation radiation. The ability to source many photons means that, despite the enormously higher attenuation of their signal by biological tissue, optical probes can provide sensitivity (i.e., the ability to detect low quantities of the probe) that is superior to that of gamma probes [2]. So while the spatial resolution possible with gamma emission imaging is much higher for subsurface sources, the sensitivity of optical imaging can be several orders of magnitude higher. The recent advent of dual gamma/optical reporter gene constructs such as HSV1-tk/luciferace [3] raises the possibility of combining the strengths of both modalities through the use of simultaneous combined modality imaging. Below, we describe the use of the localization ability of gamma emission imaging as a means of correcting for optical attenuation in fluorescence imaging.

\section{Methods And Results}

Although our ultimate goal is to develop a scanner in which CT, SPECT, and optical imaging are integrated on a single gantry, the experiments described in this paper were performed using two separate scanners. The first, which forms the basis for the ultimate tri-modality scanner, is the CT-SPECT scanner shown in Fig. 6. Since the optical component has yet to be integrated in the system, the optical measurements described here were performed using the second scanner, the Xenogen IVIS. Co-registration of the image data from the IVIS with that from the CT-SPECT scanner (and with that of the scanner of Fig. 3) was performed manually, although in the integrated system this task could be automated.

\section{GAMMA EMISSION/FLUORESCENCE STUDY}

\section{A. Attenuation Measurements}

Annexin V is a protein that is a marker for apoptosis (programmed cell death). It can be labeled with either ${ }^{99 \mathrm{~m}} \mathrm{Tc}$ or the near-IR fluorophore cyanine 5.5 (Cy5.5). In order to better understand the relative net attenuation (including absorption and scattering) of the emitted radiation from ${ }^{99 \mathrm{~m}} \mathrm{Tc}(140 \mathrm{keV})$ and Cy5.5 (peak emission wavelength of $690 \mathrm{~nm}$ ), a mouse study was performed. First, quantities of ${ }^{99 \mathrm{~m}} \mathrm{Tc}$ and Cy5.5 representative of those that would be found in a typical mouse apoptosis study using Annexin $\mathrm{V}$ were determined as follows. The estimated labeling efficiency for Cy5.5-Annexin V is two Cy5.5 


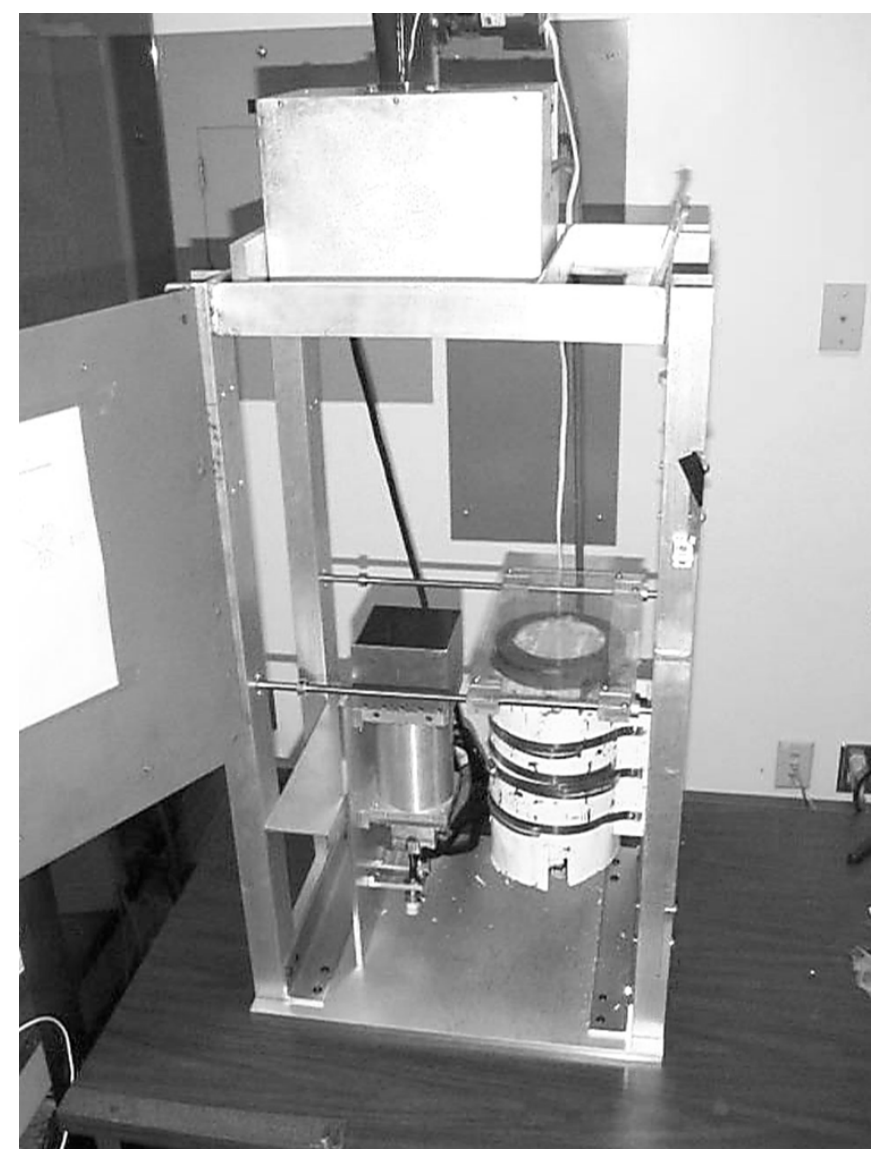

Fig. 1. Dual-modality planar x-ray (left) and gamma (right) imaging system at the University of Virginia.

molecules for every one Annexin V molecule [4]. The labeling efficiency for ${ }^{99 \mathrm{~m}} \mathrm{Tc}$ has been reported to be $\sim 95 \%$ [5]. The specific activity, defined as the activity per unit mass of the labeled protein, is dependent on many factors including the labeling efficiency and the fraction of bound $99 \mathrm{~m}$ Tc molecules that are undecayed. Values for specific activity resulting from a labeling efficiency of $\sim 95 \%$ are reported to range from $10-200 \mu \mathrm{Ci} / \mu \mathrm{g}$ [5]. This corresponds to a range in the fraction of undecayed $99 \mathrm{~m}$ Tc molecules from $0.08-1.61 \%$. For our tests, we assumed that only $0.13 \%$ of bound ${ }^{99 \mathrm{~m}} \mathrm{Tc}$ molecules were undecayed. On the average, approximately $50 \mu \mathrm{g}$ of the Annexin V protein is used in a typical study [4]. Based on the quoted relative labeling efficiencies and the assumed percentage of undecayed ${ }^{99 \mathrm{~m}} \mathrm{Tc}$ molecules present, $3.75 \mu \mathrm{g}$ of Cy 5.5 and $0.8 \mathrm{mCi}$ of $99 \mathrm{~m} \mathrm{Tc}$ were used in this study. The $99 \mathrm{~m} \mathrm{Tc}$ was first conjugated with macroaggregated albumin (MAA) to prevent diffusion throughout the animal. The $99 \mathrm{~m}$ Tc-MAA and Cy5.5 were mixed together and injected subcutaneously on the top side of a leg of a mouse. Immediately post injection, both top and bottom views of the mouse were obtained using a dualmodality planar $\mathrm{x}$-ray and gamma imaging system developed in our lab (Fig. 1) and an IVIS Imaging System 100 Series equipped with the XFO-6 Fluorescent option (Xenogen Corp. Alameda, California).

The results shown in Table I were obtained from a small ROI drawn in the center of the image of the injection site. The measured optical signal differs by over a factor of 7 depending on the
TABLE I

RESULTS FROM THE ANNEXIN V STUDY

\begin{tabular}{|c|c|c|}
\hline GAMMA & $\begin{array}{l}\text { Average counting } \\
\text { rate/pixel } \\
\text { (cps/pixel) }\end{array}$ & $\%$ change \\
\hline dorsal & 2.78 & \multirow[t]{2}{*}{1.4} \\
\hline ventral & 2.74 & \\
\hline OPTICAL & $\begin{array}{l}\text { Average pixel value } \\
\text { (ADUs) }\end{array}$ & \\
\hline dorsal & 36038 & \multirow[t]{2}{*}{86} \\
\hline ventral & 4899 & \\
\hline
\end{tabular}

Effect of Tissue Attenuation on Imaging Depth

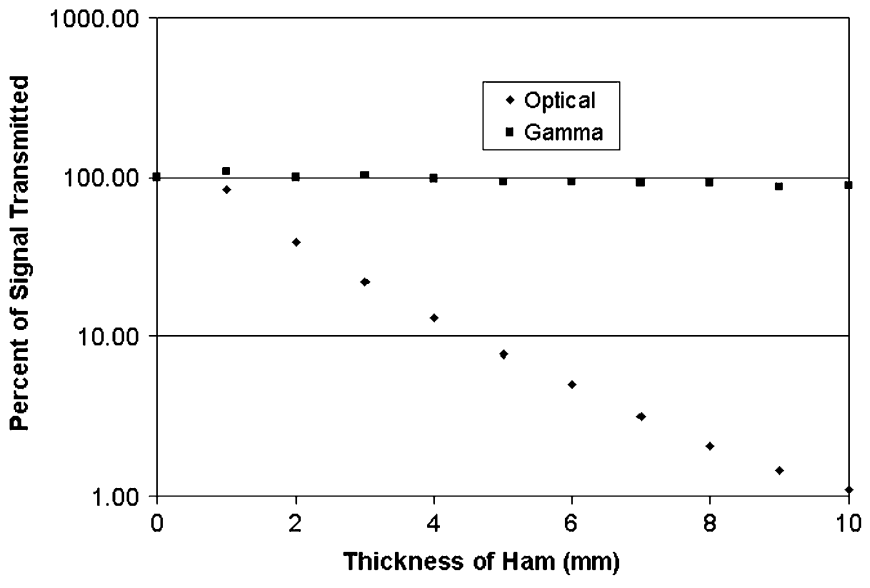

Fig. 2. Percent of signal transmitted as a function of thickness of ham for both gamma and optical imaging.

view from which the image is taken with respect to the injection site, while the measured gamma signal is nearly independent of the viewing side.

We next quantified the attenuation of the radiation emitted by $99 \mathrm{~m} \mathrm{Tc}$ and by cyanine 5.5 using a surrogate biological tissue (cooked ham). $100 \mu \mathrm{Ci}$ of ${ }^{99 \mathrm{~m}} \mathrm{Tc}$ and $50 \mu \mathrm{g}$ of the near-IR fluorophore cyanine 5.5 were placed in a spherical simulated lesion of $10 \mathrm{~mm}$ inner diameter. Varying numbers of $1 \mathrm{~mm}$ thick slices of cooked ham were placed over the lesion, and the reduction in the transmitted gamma and optical radiation was measured using a parallel hole gamma camera and the IVIS system, respectively. The reduction was determined by region of interest analysis using the resulting gamma and optical images. Integration times were held constant for both imaging systems at 3 seconds for optical and 60 seconds for gamma. The results are shown in Fig. 2. The optical signal is reduced to $1 \%$ of its original value by $10 \mathrm{~mm}$ of ham, whereas the gamma signal is only reduced to $88 \%$ of its initial level. Based on a straight line fit to the optical transmission data of Fig. 2, an approximately exponential attenuation of the optical signal is observed, with an effective linear attenuation coefficient of $\sim 0.5 \mathrm{~mm}^{-1}$. It is important to note that this attenuation coefficient takes into account both the attenuation of the excitation radiation $(615-660 \mathrm{~nm})$ and of the emitted fluorescence light. The majority of the emission light from Cy5.5 lies between 670 and $720 \mathrm{~nm}$. This is com- 


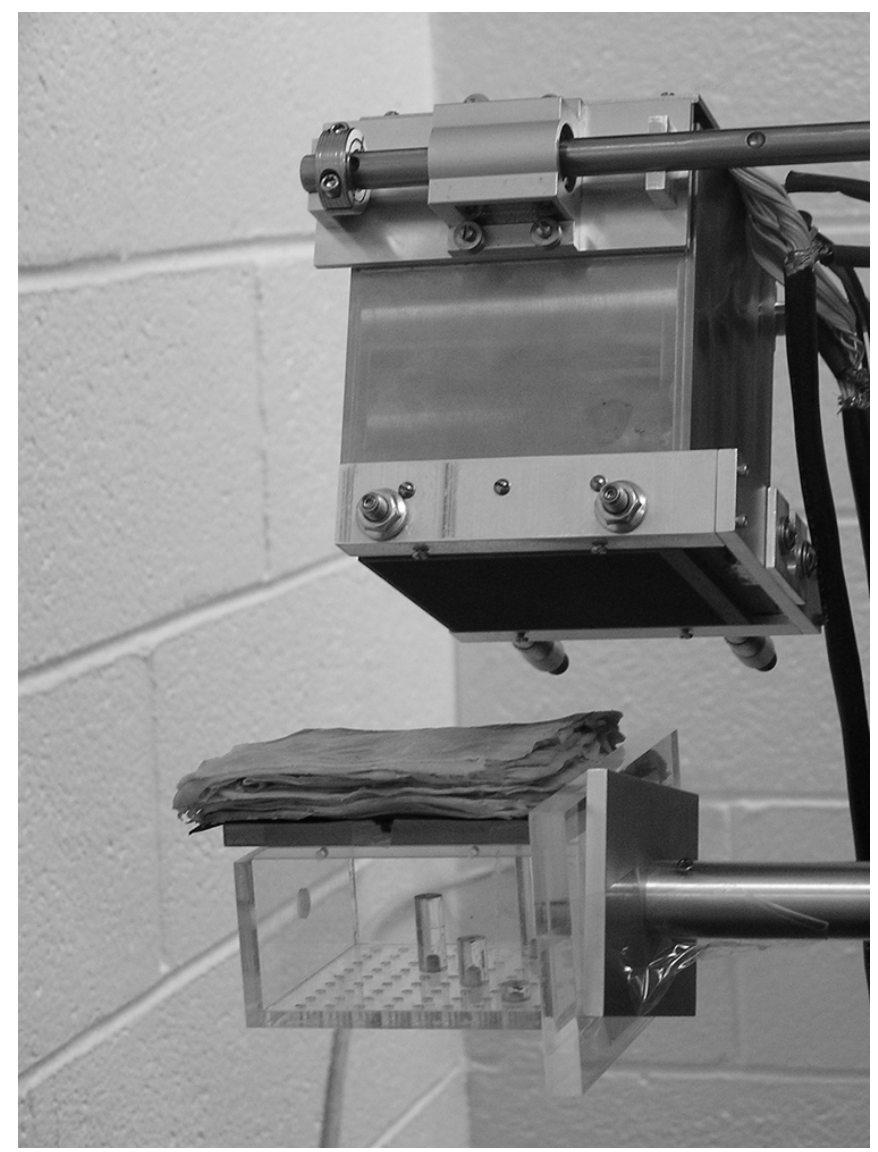

Fig. 3. Photo of stereotactic gamma emission localization. The lesion containing the ${ }^{99 \mathrm{~m}} \mathrm{Tc} / \mathrm{Cy} 5.5$ mixture is imbedded in the stack of ham.

parable to the range of 0.45 to $0.68 \mathrm{~mm}^{-1}$ reported by Zijp et al. for pig muscle with wavelengths greater than $630 \mathrm{~nm}[6]$.

It is notable that although the rate of decay of the Cy5.5 radiation in tissue is much higher than that of the gamma emission of ${ }^{99 \mathrm{~m}} \mathrm{Tc}$, the relative rates of decay of the two types of radiation in time are just the opposite. In our tests, the optical signal showed no measurable reduction in fluorescent intensity after one week, whereas the 6 hour half-life of ${ }^{99 \mathrm{~m}} \mathrm{Tc}$ resulted in a reduction in its emission intensity by a factor of $2^{28}\left(2.7 \times 10^{8}\right)$ in that time.

1) Stereotactic Gamma Localization: The goal of this experiment was to test the feasibility of using gamma ray-based source localization to make attenuation corrections to the measured optical signal. The mixing protocol for Cy5.5 and ${ }^{99 \mathrm{~m}} \mathrm{Tc}$ was kept the same as described in the above section i.e., $3.75 \mu \mathrm{g}$ of Cy 5.5 and $0.8 \mathrm{mCi}$ of ${ }^{99 \mathrm{~m}} \mathrm{Tc}$. The mixture was injected in a $4 \mathrm{~mm}$ inner diameter lesion and embedded in a phantom made of cooked ham at various depths. For each source depth, a fluorescence image was obtained using the IVIS Xenogen optical camera operated in the fluorescence mode. The light signal was characterized by measuring the average pixel value in a small region of interest (ROI) centered on the image of the lesion. Then, for each source depth, the phantom was imaged stereotactically using the gamma camera shown in Fig. 3. Two gamma emission images were obtained, separated by 40 degrees. The difference in the imaged location of the lesion in the two views was used to

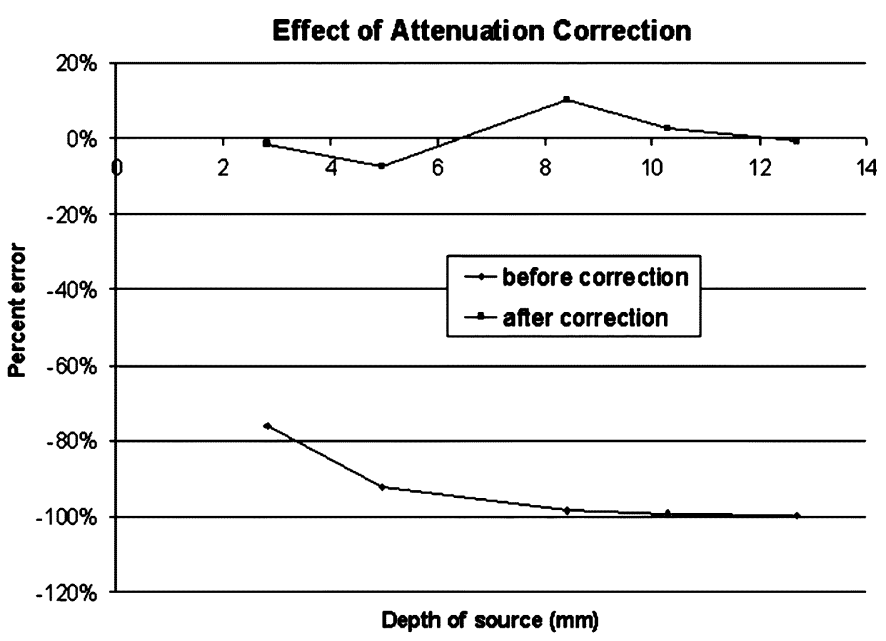

Fig. 4. Effect of attenuation correction using multiple view gamma imaging. Note that $-100 \%$ error corresponds to zero light signal.

calculate the lesion depth within the phantom. Using each calculated depth and the optical attenuation coefficient measured in A.1., the measured ROI pixel value in the corresponding optical image was corrected as follows:

$$
P_{\text {corr }}=P_{\text {raw }} \exp \left(\mu_{\text {eff }} t\right)
$$

where $P_{\text {corr }}$ the corrected pixel value, $P_{\text {raw }}$ is the uncorrected pixel value, $\mu_{\mathrm{eff}}$ is the effective attenuation coefficient and $t$ is the calculated ham thickness. The error in the measured pixel value (compared to that measured with zero attenuation) before and after correction is shown in Fig. 4. Note that $-100 \%$ error corresponds to zero light signal.

\section{B. CT/Bioluminescence Study}

In situations where the optical emission originates from a specific anatomical site (for example from a tumor or from a specific organ), it may be possible to use CT to aid in attenuation correction of the measured optical signal. To test this, we performed a CT/bioluminescence phantom study. The bioluminescence source was created using the ATP-lite 1 step bioluminescent kit from Perkin Elmer. The kit is primarily intended as an ATP monitoring assay based on luciferin, luciferase and ATP. A small quantity of the luciferace/luciferin/ATP mixture was first used to measure the attenuation of luciferace light by the surrogate biological tissue. The results are shown in Fig. 5. Although a simple single exponential model for the net attenuation is not as good an approximation as it was in the case of the fluorescence study above, a best fit line to the data of Fig. 5 yields an attenuation coefficient of $0.42 \mathrm{~mm}^{-1}$.

A phantom was prepared with $1 \mu \mathrm{M}$ (80 ul) of the luciferace assay and combined with $40 \mu \mathrm{l}$ of gadolinium x-ray contrast. $100 \mathrm{ul}$ of the mixture was injected into a small simulated breast lesion (4 mm inner diameter) and embedded in a cylindrical ham phantom, $\sim 2.4 \mathrm{~cm}$ diameter. The phantom was placed in an acrylic cylinder of 1 " inner diameter and $0.7 \mathrm{~mm}$ wall thickness. The phantom was rotated in steps of 45 degrees over 360 degrees (8 views) and the transmitted light out was measured using the IVIS Xenogen camera. The same phantom was then scanned in the CT-SPECT scanner, which was equipped 


\section{Linear attenuation coefficient of ham for luciferase emission}

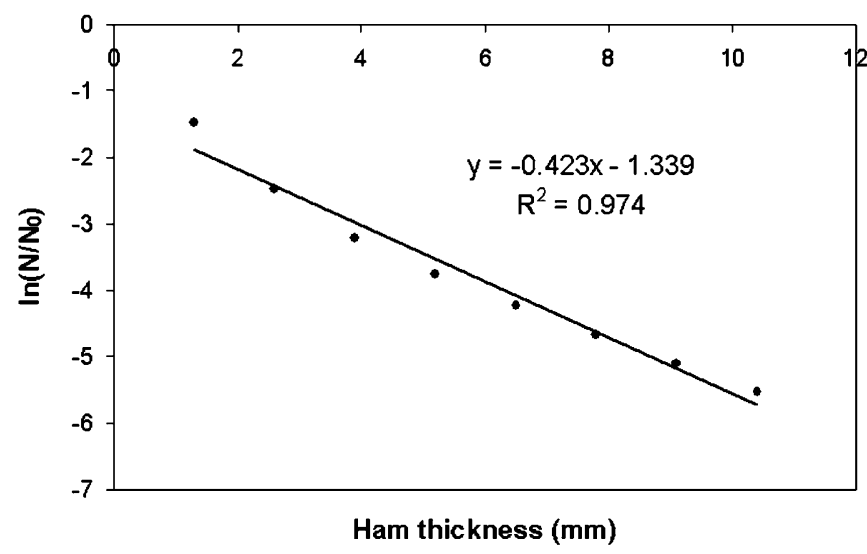

Fig. 5. Measured attenuation of luciferace light by cooked ham.

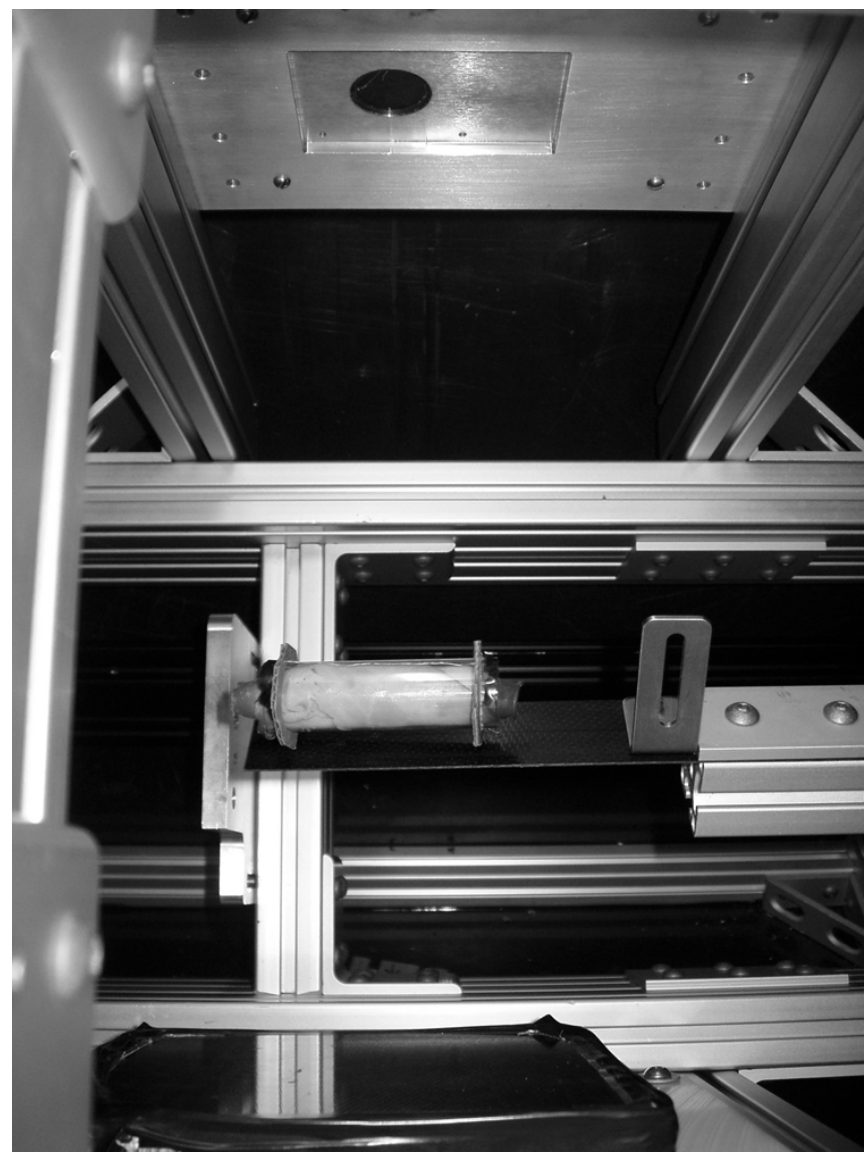

Fig. 6. Photo of the phantom being CT scanned in the tri-modality scanner.

with a Hamamatsu model C7940DA-02 x-ray detector and a Source-ray x-ray source (model SB-40-1 k, Source-ray Inc., Bohemia, NY). The scan was over 360 degrees, with 0.72 degree increments (500 views). A photo of the phantom inside the scanner gantry is shown in Fig. 6. To attenuation correct the light output from the optical camera, an axial CT slice (Fig. 7) through the lesion was used to determine, for each view, the total thickness of the ham between the lesion and the phantom surface. Air gaps within the phantom were identified in the CT image, and were subtracted from the total distance from the

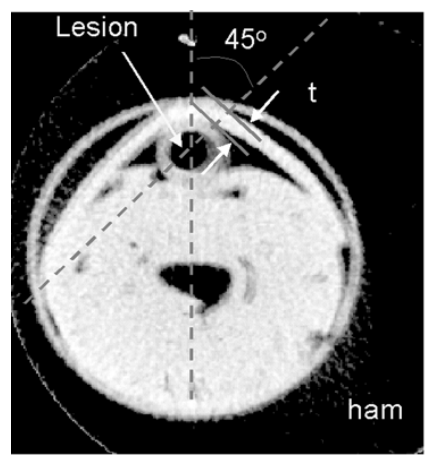

Fig. 7. Axial slice from the phantom CT scan. The thickness of ham attenuating the luciferace emission in the 45 degree view is denoted in the figure as $\mathrm{t}$.

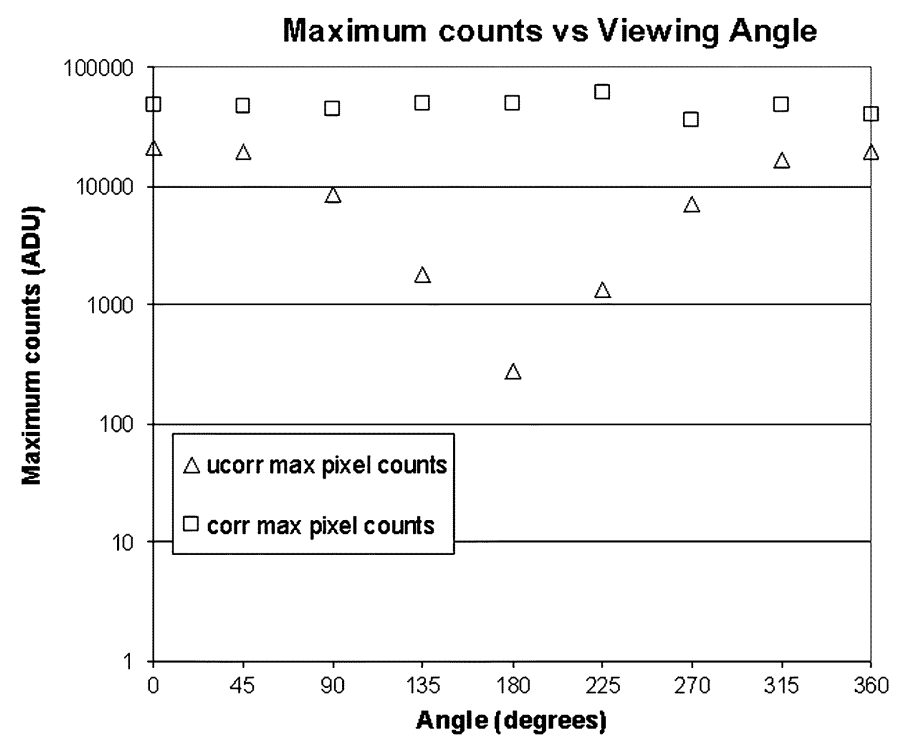

Fig. 8. Maximum pixel value in an ROI centered on the optical image of the lesion, before (triangles) and after (squares) attenuation correction.

lesion center to the surface of the phantom to arrive at the total ham thickness. Correction to the ROI pixel values in the optical images were performed according to (1). Fig. 8 shows the pixel values before and after correction. The corrected values clearly show less dependence on the viewing angle compared to the uncorrected values.

\section{CONCLUSION}

Our preliminary tests suggest that it may be possible to use gamma emission or $\mathrm{x}$-ray transmission information to improve estimates of light output from fluorescence or bioluminescence sources. The single exponential model used here is certainly only approximate, and provides only a rough attenuation correction. Accurate quantification of the optical signal will require careful modeling of the depth dependent hardening of the transmitted beam. However, even this overly simplified model results in significant improvement. Further tests are needed to validate this approach for in vivo imaging, where the optical attenuation paths are less homogeneous, and reflection from tissue boundaries within the animal could be significant.

Multi-modality imaging also could prove valuable for more elaborate approaches to in vivo optical imaging. For example, 
the modeling of light diffusion through turbid media has been undertaken by several groups [7], [8]. In the application of such models to the problem of predicting the internal distribution of optical sources based on the measured light distribution at the surface, typically the assumed internal source distribution is iteratively adjusted based on the discrepancy between the measured light distribution at the surface and that predicted using the forward model. The use of a complimentary imaging modality like CT could enable the inclusion in the model of spatial variation in the absorption and scattering coefficients based on the imaged locations of various tissue types.

Recent studies by Troy et al. [9] have shown that by determining the 3-D surface topography of the animal and acquiring a series of images at varying wavelengths between 560 and $660 \mathrm{~nm}$ (Xenogen, IVIS 200), the depth and the intensity of light sources inside a living animal can be estimated using a diffusion model for light propagation in tissue [10]. Encouraging results have been obtained using a homogeneous tissue model as a first approximation, however, the authors state that the largest source of error is uncertainty in the optical properties of the tissue, and thus the next phase is to incorporate a heterogeneous tissue model. Structural image data such as that available via CT could permit improved source intensity estimates through better characterization of the optical path to the surface.

\section{ACKNOWLEDGMENT}

The authors would like to thank Y. Zheng and H. Li of the Electrical and Computer Engineering Department at the University of Virginia for help with CT reconstruction.

\section{REFERENCES}

[1] L. Celentano et al., "Preliminary tests of a prototype system for optical and radionuclide imaging in small animals," IEEE Trans. Nucl. Sci., pt. 2, vol. 50, no. 5, pp. 1693-1701, Oct. 2003.

[2] T. Troy et al., "Quantitative comparison of the sensitivity of detection of fluorescent and bioluminiscent reporters in animal models," Molecular Imag., vol. 3, pp. 9-23, Jan. 2004.

[3] P. Ray et al., "Optical bioluminescence and positron emission tomography imaging of a novelfusion reporter gene in tumor xenografts of living mice," Cancer Res., vol. 63, pp. 1160-1165, Mar. 2003.

[4] D. Pan et al., "Synthesis and characterization of Cyanine 5.5-annexin V, an Apoptosis imaging agent," presented at the 2nd Annu. Meeting Soc. Molecular Imaging, San Francisco, CA, Aug. 2003. Abstract 107.

[5] F. Blankenberg et al., "In-vivo detection and imaging of phosphatidylserine expression during programmed cell death," in Proc. Nat. Acad. Sci., vol. 95, May 1998, pp. 6349-6354.

[6] J. Zijp et al., "Optical properties of bovine muscle tissue in-vitro; a comparison of methods," Phys. Med. Biol., vol. 43, pp. 3065-3081, Jul. 1998.

[7] S. R. Arridge, "Optical tomography in medical imaging," Inverse Problems, vol. 15, no. 2, pp. 41-93, 1999.

[8] A. D. Klose et al., "The inverse source problem based on the radiative transfer equation in optical molecular imaging," J. Comput. Phys., vol. 202, pp. 323-345, 2005.

[9] T. Troy et al., "Single-view bioluminiscent tomography in small animal models," presented at the 4th Soc. Molecular Imaging Conf., Cologne, Germany, Sep. 2005. Paper 513.

[10] B. W. Rice et al., "In-vivo imaging of light emitting probes," J. Biomed. Opt., vol. 6, no. 4, pp. 432-440, Oct. 2001. 